\title{
ASPECTOS BIOLÓGICOS DE Holochilus sp., HOSPEDEIRO NATURAL DA ESQUISTOSSOMOSE
}

\section{BIOLOGICAL ASPECTS OF RODENT Holochilus sp., NATURAL HOST OF SCHISTOSOMIASIS}

\author{
Maria Gabriela Sampaio Lira ${ }^{1^{*}}$ \\ Guilherme Silva Miranda ${ }^{1}$ \\ João Gustavo Mendes Rodrigues ${ }^{1}$ \\ Ranielly Araújo Nogueira ${ }^{1}$ \\ Gleycka Cristine Carvalho Gomes ${ }^{1}$ \\ Lorrane Gabrielle Cantanhêde ${ }^{1}$ \\ Nêuton Silva-Souza ${ }^{1}$
}

${ }^{1}$ Universidade Estadual do Maranhão, São Luis, MA, Brasil.

*Autora para correspondência - gabrielasampaiolira@yahoo.com.br

\section{Resumo}

Neste estudo objetivou-se analisar os aspectos reprodutivos (período estacional e quantidade de embriões) e a positividade para $S$. mansoni em roedores do gênero Holochilus, no município de São Bento - MA. Para isso, foram realizadas capturas mensais de até 10 roedores Holochilus sp., aleatoriamente quanto ao sexo, anotando-se o período estacional do município de São Bento nos meses de captura. Em laboratório, os roedores tiveram as fezes analisadas quanto à positividade para S. mansoni pelo método Kato-Katz. Após a análise, os animais foram anestesiados para a obtenção do comprimento total, peso corporal e determinação do sexo. Nas fêmeas, foi realizada a incisão da porção ventral do corpo, para a verificação da presença de embriões e contagem dos mesmos. Dentre os roedores capturados, ocorreu dominância de machos em quase todos os meses e eles tenderam a ser mais pesados e apresentaram maior porte que as fêmeas. Os animais mostraram elevado potencial reprodutivo. A positividade para S. mansoni e a reprodução dos Holochilus sp. foram maiores no período chuvoso de São Bento. Quanto ao número de embriões, constatou-se que, no período chuvoso, foram encontrados em maior quantidade por fêmea. Em suma, a intensa atividade reprodutiva juntamente à suscetibilidade para $S$. mansoni apresentada pelos roedores são fatores que ajudam na manutenção e agravam a esquistossomose na Baixada Maranhense.

Palavras-chave: roedor; reprodução; Schistosoma mansoni.

\begin{abstract}
In the present study, we aimed to analyze the reproductive aspects (seasonal period and number of embryos) and positivity for $S$. mansoni in rodent of Holochilus gender, in São Bento - MA. For this, monthly captures of up to 10 Holochilus sp. rodents were conducted, randomly for sex, taking notes of the seasonal period of São Bento in each month of capture. At the laboratory, rodents had feces analyzed for $S$. mansoni infection by Kato-Katz method. After the analysis, they were adequately anesthetized for obtaining full length, weight and sex determination. In females, an incision of the ventral portion of the body was carried out to verify the presence of embryos and to count them. Among the captured rodents, there was male dominance in almost all months and they tended to be heavier and had larger bodies than the females. The animals showed high reproductive potential. The positivity for $S$. mansoni and the reproduction of Holochilus sp. were greater in the rainy season of
\end{abstract}


São Bento. As for the number of embryos, we observed that, in the rainy season, more males were found than female. In short, the intense reproductive activity, along the susceptibility to $S$. mansoni presented by rodents are factors that help to maintain and aggravate schistosomiasis in Baixada Maranhense.

Keywords: rodent; reproduction; Schistosoma mansoni.

Enviado em: 10 agosto 2015

Aceito em: 02 dezembro 2015

\section{Introdução}

A esquistossomose mansônica é uma doença parasitária causada pelo helminto Schistosoma mansoni, que acomete pacientes do mundo inteiro(1). No Brasil, estima-se que 25 milhões de pessoas vivam em áreas endêmicas, sendo o maior número de casos da enfermidade notificados nos estados do Nordeste, devido ao deficiente saneamento básico e má higienização sanitária da população ${ }^{(2,3)}$.

É provável que esquistossomas parasitos de roedores (S. rodahini) associados à evolução dos primeiros hominídeos tenham dado origem ao $S$. mansoni $i^{(4,5)}$. Com isso, posteriormente, o parasito passou a infectar também os roedores silvestres ${ }^{(5)}$. O ciclo biológico silvestre do $S$. mansoni, que tem como hospedeiro definitivo os roedores, equivale ao ciclo tradicional, que tem o ser humano como hospedeiro definitivo, pois ocorrem os mesmos processos desde a infecção no hospedeiro intermediário (molusco do gênero Biomphalaria) até o hospedeiro definitivo ${ }^{(6)}$. Dessa forma, a presença de populações de roedores silvestres naturalmente infectados pelas cercárias de $S$. mansoni, que são eliminadas na água pelos caramujos do gênero Biomphalaria, tornou-se um fator complicador para os programas de controle ${ }^{(6)}$.

Ao se considerar a vasta distribuição geográfica ${ }^{(7,8)}$, a ocorrência de animais infectados em praticamente todas as áreas endêmicas onde foram investigados ${ }^{(9)}$, a tolerância à presença humana, ocorrendo no peri-domicílio, e os hábitos semi-aquáticos, fator preponderante para a infecção da esquistossomose, os roedores dos gêneros Nectomys e Holochilus destacam-se como potenciais reservatórios naturais do $S$. mansoni quando comparados a diversas outras espécies de roedores silvestres encontrados parasitados no Brasil (Oxymycterus sp., Necromys lasiurus, Akodon spp., Sooretamys spp., Calomys spp., Proechimys sp., Rattus rattus, Rattus norvegicus e Cavia aperea $)^{(10,11)}$.

Na Baixada Ocidental Maranhense, Bastos et al. ${ }^{(12)}$ constataram a coabitação de dois hospedeiros definitivos do S. mansoni: o ser humano e o roedor silvestre do gênero Holochilus. Hoje, sabe-se que o roedor Holochilus sp. é um importante elo da cadeia epidemiológica do S. mansoni, funcionando como um possível reservatório natural, não só por albergar grande número de parasitos adultos, mas também por eliminar ovos maduros em suas fezes durante todo o ano, destacando-se por serem importantes na manutenção da helmintíase na região ${ }^{(6,13)}$. Além disso, a Baixada Maranhense é considerada como uma área endêmica para a esquistossomose, caracterizando-se também pela falta de saneamento básico adequado, concentração populacional e coleções hídricas contendo grande número de caramujos suscetíveis à infecção, com predomínio das espécies Biomphalaria glabrata e Biomphalaria straminea ${ }^{(14)}$.

Os espécimes de roedores do gênero Holochilus são animais terrestres, predominantemente noturnos, de hábitos semi-aquáticos, dotados de pequenas membranas interdigitais que auxiliam na locomoção dentro d'água, sendo, portanto, perfeitamente capazes de se adaptarem à ecologia da Baixada Maranhense, a qual apresenta um período estacional seco e um chuvoso ${ }^{(15)}$.

O ciclo reprodutivo de Holochilus sp. é semelhante ao de outros pequenos mamíferos da região tropical, e se sabe que o período fértil das fêmeas dura cerca de quatro dias e os animais estão aptos para o acasalamento com três meses de vida. A gestação dura em média 21 dias com aproximadamente 5 a 10 filhotes por ninhada ${ }^{(8,6)}$. Os roedores costumam fazer ninhos em restos de madeira ou, principalmente, nos empilhamentos entre as linhas da vegetação aquática, onde se protegem dos predadores e costumam construir seus ninhos elipsoidais, com média de 22,5 cm de comprimento, 13 $\mathrm{cm}$ de largura e $18,7 \mathrm{~cm}$ de altura ${ }^{(16)}$.

Tendo em vista a efetiva capacidade dos roedores do gênero Holochilus de funcionarem como 
hospedeiros definitivos da doença, é de muita importância conhecer também outros aspectos da biologia desse animal, visto que uma acelerada reprodução associada à manutenção do ciclo do parasito em uma área endêmica aumenta a probabilidade de existirem mais potenciais reservatórios da esquistossomose. Nesse sentido, o trabalho teve por objetivo analisar parâmetros biológicos (período estacional reprodutivo e quantidade de embriões) em roedor do gênero Holochilus, no município de São Bento - MA, relacionando também com a positividade dos animais silvestres para $S$. mansoni, de forma a auxiliar ações de controle da enfermidade na localidade e contribuir com outras pesquisas nessa área.

\section{Material e Métodos}

O estudo foi desenvolvido no município de São Bento, localizado na Baixada Ocidental Maranhense, que situa-se a uma latitude $02^{\circ} 41^{\prime} 45^{\prime \prime}$ Sul e a uma longitude $44^{\circ} 49^{\prime} 17^{\prime}$ ' Oeste, estando a uma altitude de 2 metros. A cidade apresenta clima tropical úmido, onde são perceptíveis dois períodos estacionais, o chuvoso, entre janeiro e julho, e o seco entre agosto e dezembro.

Mensalmente, realizou-se a captura de roedores no município de São Bento - MA, no período de agosto de 2013 a julho de 2014, de forma totalmente aleatória quanto ao sexo. Para captura dos roedores, foram utilizadas armadilhas do tipo Tomahawk, as quais foram depositadas em alguns pontos do campo, característico da região de São Bento. As armadilhas foram colocadas durante a noite, obedecendo a uma distância mínima de 10 metros uma da outra contendo isca de banana untada com pasta de amendoim. Os roedores capturados foram então, conduzidos ao laboratório da Fazenda Escola no Campus da Universidade Estadual do Maranhão de São Bento - MA.

Posterior à captura, as fezes dos animais foram analisadas pelo método de Kato-Katz ${ }^{(17)}$, de forma qualitativa, para comprovar a positividade para S. mansoni. Nesse processo, foram utilizadas três lâminas para cada amostra fecal e o número de roedores positivos foi então anotado, verificandose diferenças entre o período seco e chuvoso da cidade de São Bento - MA. Todas as etapas, desde a captura até a experimentação dos roedores, foram autorizadas pelo Instituto Brasileiro de Meio Ambiente - IBAMA, de acordo com a licença para atividades com finalidades científicas n40025/1, e está devidamente protocolado no Conselho de Ética e Experimentação Animal da Universidade Estadual do Maranhão, com o número 06/2014.

Foram realizadas as análises biométricas dos roedores, fazendo-se o uso dos anestésicos ketamina 5\% e cloridrato de xylasina $2 \%$, sendo utilizados 0,1 e $0,2 \mathrm{~mL}$ por $100 \mathrm{~g}$ de peso, e administrados por via intraperitoneal. Com os roedores devidamente anestesiados, foi utilizada a fita métrica para medir o comprimento total (CT) e, com o auxílio da balança, obteve-se o peso corporal (PC). Além disso, os animais capturados foram todos identificados quanto ao sexo.

Com os roedores diferenciados quanto ao sexo e anestesiados, foi realizada, apenas nas fêmeas, incisão com tesoura cirúrgica da porção ventral-caudal do corpo, seguindo a linha mediana, partindo da região pubiana no sentido da porção divisória entre a cavidade abdominal e a caixa torácica. Para a coleta dos embriões separaram-se os cornos uterinos, e procedeu-se com a contagem da quantidade de embriões nessa estrutura por fêmeas prenhes. Posteriormente, realizou-se a eutanásia nas fêmeas ${ }^{(18)}$. Ademais, foram anotadas a quantidade de roedores fêmeas prenhes encontradas mensalmente, verificando-se diferenças, em quantidade, entre o período seco e chuvoso, para determinar os meses de maior intensidade reprodutiva.

Para se verificar a existência de diferenças significativas na proporção entre os sexos, foi utilizado o teste do $\chi^{2}$ com correção de Yates com nível de significância de 5\%. Verificou-se também a relação entre comprimento total e peso corporal por meio da regressão não linear. $\mathrm{O}$ ajuste da curva representada pela expressão matemática $\mathrm{PC}=\mathrm{a} \times \mathrm{CT}^{\mathrm{b}}$, foi obtida pelo método dos mínimos quadrados ${ }^{(19)}$, em que PC é o peso corporal do roedor, a é o coeficiente linear de regressão, CT é o comprimento total do roedor e b é o coeficiente angular de regressão. Após a análise, foi possível classificar o crescimento nas relações comprimento total x peso corporal em: $\mathrm{b}<3$, alométrico negativo, ou seja, a variável dependente $(\mathrm{Y})$ cresce a uma taxa relativamente menor que a independente $(\mathrm{X}) ; \mathrm{b}>3$, alométrico positivo, a variável independente $(\mathrm{Y})$ cresce em uma taxa relativamente maior que a independente 
$(\mathrm{X})$; e $\mathrm{b}=3$, crescimento isométrico, as partes morfométricas ( $\mathrm{X} \mathrm{e} \mathrm{Y}$ ) crescem de maneira uniforme ${ }^{(20)}$.

\section{Resultados e Discussão}

Considerando-se o período de amostragem (agosto de 2013 a julho de 2014) e a quantidade de até 10 roedores capturados nos meses do ano em estudo, a proporção de indivíduos quanto ao sexo observada para o período total, foi mais favorável aos machos $(1.62 \mathrm{M}: 1.00 \mathrm{~F} ; \mathrm{p}<0.05)$, ocorrendo dominância deles em quase todos os meses de captura.

Graipel et al. ${ }^{(21)}$ encontraram resultado semelhante de quantidade de indivíduos quanto ao sexo, quando observaram dominância na proporção de machos sobre as fêmeas para várias espécies de pequenos mamíferos, entre elas, de roedores. A proporção sexual desviada para os machos está relacionada, segundo muitos autores, aos maiores deslocamentos que eles realizam, o que é considerado um padrão entre os mamíferos ${ }^{(22,23)}$. No entanto, D'Andrea et al. ${ }^{(24)}$ afirmaram que essa razão do sexo desviada para machos entre muitas espécies de roedores de hábito semi-aquático não é, provavelmente, uma realidade biológica, apesar de já ter sido reportada na literatura. Segundo eles, este desvio deve ser proveniente de uma densidade maior de machos em cada trecho de rio/campo em que foram depositadas as armadilhas, mas não na área de abrangência da população como um todo. Esta diferença na densidade por trecho de rio/campo seria uma consequência dos machos sobreporem suas áreas de utilização mais intensamente, ao passo que as fêmeas não as sobrepõem da mesma forma ${ }^{(24)}$, sendo, portanto, necessária a expansão do espaço de captura para a obtenção da mesma quantidade de roedores de ambos os sexos.

O valor total de $\chi^{2}$ encontrado $(5,95 ; \mathrm{p}<0,05)$ confirmou a diferença na proporção de indivíduos quanto ao sexo durante as capturas realizadas no período de um ano, tendo sido encontradas distinções maiores para os meses de setembro e julho (Tabela 1)

Tabela 1: Distribuição da frequência absoluta ( $\mathrm{Fi})$, frequência esperada ( $\mathrm{Fe}$ ) e valores do quiquadrado $\left(\chi^{2}\right)$ de roedores machos e fêmeas, em cada mês de captura

\begin{tabular}{cccccc}
\hline Meses & Machos (Fi) & Fêmeas (Fi) & Machos + Fêmeas & Fe & $\chi^{\mathbf{2}}$ \\
\hline Ago/13 & 6 & 2 & 8 & 4 & $\mathbf{2}$ \\
Set/13 & 8 & 0 & 8 & 4 & $\mathbf{8}^{*}$ \\
Out/13 & 7 & 3 & 10 & 5 & $\mathbf{1 , 6}$ \\
Nov/13 & 5 & 5 & 10 & 5 & $\mathbf{0}$ \\
Dez $/ 13$ & 6 & 1 & 7 & 3,5 & $\mathbf{3 , 5 7 1 4 2 9}$ \\
Jan/14 & 8 & 2 & 10 & 5 & $\mathbf{3 , 6}$ \\
Fev/14 & 7 & 3 & 10 & 5 & $\mathbf{1 , 6}$ \\
Mar/14 & 8 & 2 & 10 & 5 & $\mathbf{3 , 6}$ \\
Abr/14 & 2 & 8 & 10 & 5 & $\mathbf{3 , 6}$ \\
Mai/14 & 5 & 4 & 9 & 4,5 & $\mathbf{0 , 1 1 1 1 1 1}$ \\
Jun/14 & 3 & 4 & 7 & 3,5 & $\mathbf{0 , 1 4 2 8 5 7 1 4 3}$ \\
Jul/14 & 0 & 6 & 6 & 3 & $\mathbf{6}^{*}$ \\
TOTAL & 65 & 40 & 105 & 52,5 & $\mathbf{5 , 9 5 2 3 8 0 9 5 2 *}$ \\
\hline
\end{tabular}

${ }^{*}$ valores $\chi^{2}$ que revelaram diferenças na proporção de indivíduos quanto ao sexo. 
Os machos apresentaram porte maior que as fêmeas, com a maioria deles sendo encontrados na classe de comprimento de $33-36 \mathrm{~cm}$. Enquanto que a maior quantidade das fêmeas obteve comprimento que variou entre 27 a $30 \mathrm{~cm}$, não sendo verificada nenhuma fêmea nas classes de comprimento de 36-39 e $39-41 \mathrm{~cm}$ (Figura 1).

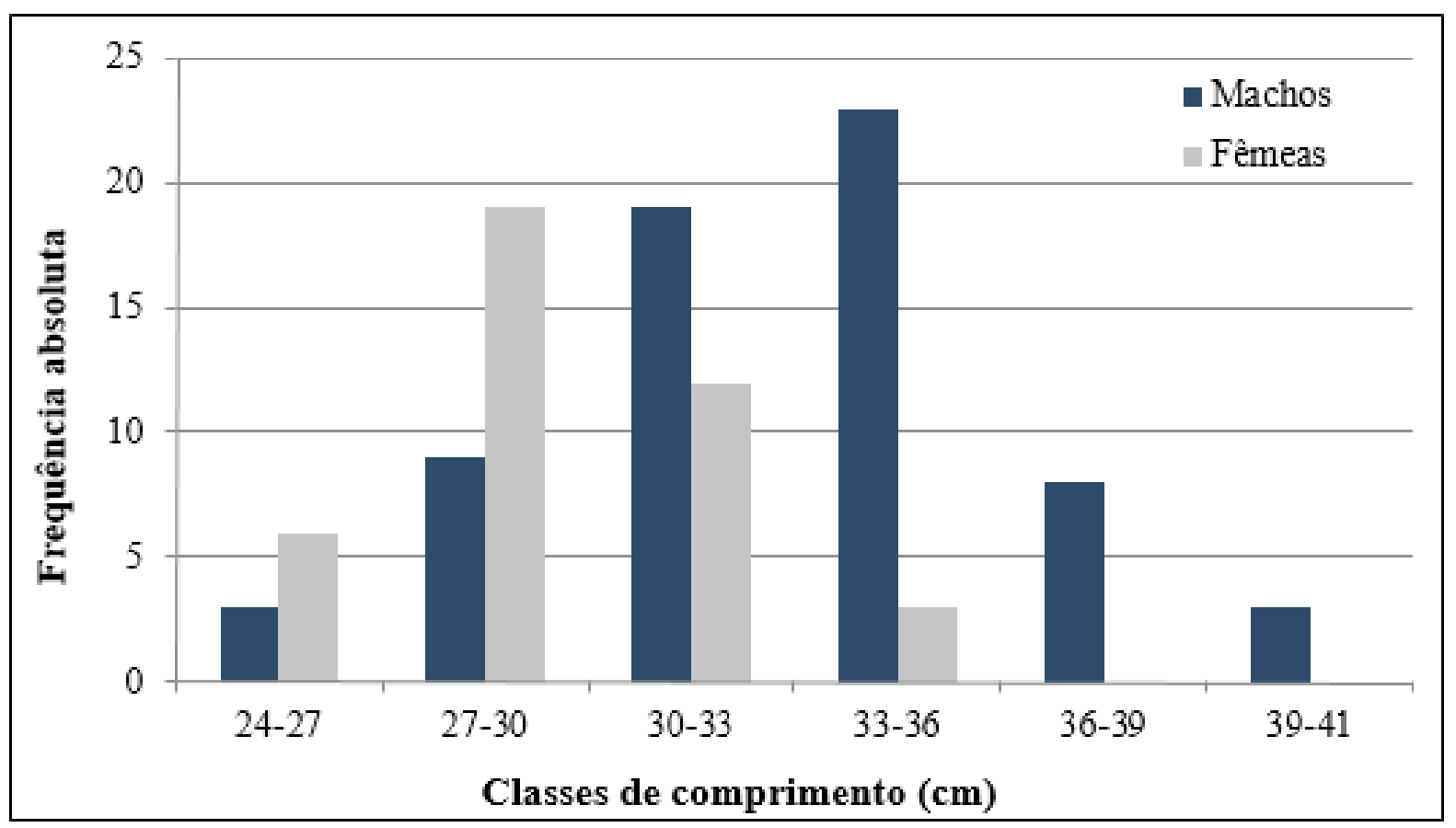

Figura 1: Classes de comprimento de roedores silvestres, machos e fêmeas, capturados em Bento - MA.

Com relação ao peso corporal, as fêmeas tenderam a ser bem menores que os machos, sendo o peso mínimo apresentado pelos machos de $107,7 \mathrm{~g}$ e máximo de $320 \mathrm{~g}$, enquanto que o mínimo das fêmeas foi de $97,12 \mathrm{~g}$ e o máximo de $230 \mathrm{~g}$; com isso, pode-se dizer que naturalmente as fêmeas são mais leves do que os machos, percebendo-se uma elevação de peso durante a gestação, principalmente se a quantidade de embriões que elas carregam for grande ( $>4$ embriões). A disponibilidade de alimento, o ambiente, a genética da espécie ou até mesmo atividades diferenciadas realizadas de acordo com o sexo do animal são possíveis fatores responsáveis pela distinção entre peso e comprimento para machos e fêmeas desses roedores ${ }^{(25)}$.

Estudos mostram que em muitas espécies de roedores silvestres da subfamília Signomodontinae, precisamente da tribo Oryzomyini, semelhantes aos Holochilus sp., os machos tendem a ser maiores e mais pesados do que as fêmeas, sendo um dos motivos citados para este acontecimento, a ocorrência de um sistema de acasalamento promíscuo ${ }^{(26,27)}$. Segundo D'Andrea et al. ${ }^{(28)}$, os machos e fêmeas de roedores dessa subfamília não diferem em peso e comprimento ao nascerem; entretanto, os machos, com o passar do tempo, adquirem um crescimento mais acelerado e ficam mais pesados para a obtenção de sucesso no acasalamento com mais de uma fêmea.

Quanto à correlação peso x comprimento, contatou-se, a partir dos valores obtidos para o coeficiente de regressão angular, que a alometria foi negativa para machos $(b=0,2365)$, fêmeas $(b=0,2817)$ e sexos agrupados $(b=0,2399)$, o que significa dizer que os roedores do gênero Holochilus tendem a crescer mais em comprimento do que em peso no decorrer da sua vida. Apesar da alometria negativa, em um estudo de observação do crescimento de roedores do gênero Holochilus em laboratório, realizado por Kawazoe e Pinto ${ }^{(29)}$, verificou-se que à medida que o roedor cresce, seu peso aumenta e este aumento é bastante homogêneo quando se comparam diferentes exemplares, discordando do resultado encontrado neste trabalho ou indicando que o crescimento maior em comprimento do que 
em peso é minimamente perceptível ou não perceptível.

No que diz respeito aos parâmetros reprodutivos, foram capturadas um total de 40 roedores fêmeas, dessas, 27 encontraram-se prenhes. As fêmeas prenhes foram obtidas em quase todos os meses em que houveram fêmeas capturadas, exceto em dezembro e janeiro; no entanto, nesses mesmos meses, a quantidade de fêmeas encontradas foi pequena $(\leq 2)$.

É importante ressaltar que, nos meses de maio, junho e julho, todas as fêmeas encontradas estavam prenhes, indicando que estes meses correspondem ao tempo de maior intensidade reprodutiva dos roedores Holochilus sp., concordando com Veiga-Borgeaud et al. ${ }^{(13)}$, que afirmaram que o período compreendido de março a julho é considerado de intensa reprodução para esses animais. Apesar disso, resultados encontrados para reprodução dos roedores do gênero Holochilus em cativeiro, verificados por Kawazoe e Pinto ${ }^{(29)}$, parecem indicar que em ambiente favorável, com espaço suficiente para a sobrevivência dos roedores e alimentação em abundância, não há época marcada para a sua reprodução. Quanto ao número de embriões, observou-se que no período seco (agosto a dezembro) do município de São Bento, apenas no mês de novembro, foi encontrada uma grande quantidade de embriões nas fêmeas prenhes, variando entre oito e nove, enquanto que no período chuvoso (janeiro a julho) do munícipio, este número cresceu significativamente chegando a serem encontrados 12 embriões em uma única fêmea (Tabela 2). Esse número é bastante alto, levando-se em consideração que esses animais são pequenos mamíferos, mas está em proximidade com a quantidade de filhotes por ninhada conhecida para esse gênero de roedor (até 10 filhotes) ${ }^{(8)}$. Esse dado corrobora também com o fato de que esses roedores apresentam uma elevada densidade populacional na Baixada Maranhense ${ }^{(13)}$.

Tabela 2: Quantidade de embriões encontrados em cada mês, no município de São Bento - MA.

\begin{tabular}{ccc}
\hline $\begin{array}{c}\text { Meses do ano } \\
(\mathbf{2 0 1 3} / \mathbf{2 0 1 4})\end{array}$ & Fêmeas prenhes & Número de embriões \\
\hline $\mathrm{Ago} / 13$ & 1 & 4 \\
$\mathrm{Set} / 13$ & 0 & 0 \\
$\mathrm{Out} / 13$ & 1 & 4 \\
$\mathrm{Nov} / 13$ & 2 & $8-9$ \\
$\mathrm{Dez} / 13$ & 0 & 0 \\
$\mathrm{Jan} / 14$ & 0 & 0 \\
$\mathrm{Fev} / 14$ & 1 & 6 \\
$\mathrm{Mar} / 14$ & 1 & 4 \\
$\mathrm{Abr} / 14$ & 7 & $3-7$ \\
$\mathrm{Mai} / 14$ & 4 & $5-6$ \\
$\mathrm{Jun} / 14$ & 4 & $6-9$ \\
$\mathrm{Jul} / 14$ & 4 & $7-12$ \\
\hline
\end{tabular}

Neste contexto, constatou-se que o período chuvoso (janeiro a julho) de São Bento - MA correspondeu ao tempo de maior reprodução dos roedores do gênero Holochilus, como mostra a figura abaixo (Figura 2), pois nos meses desse período foi encontrado o maior número de fêmeas prenhes e também uma maior quantidade de embriões. Este dado também está de acordo com um trabalho anteriormente executado por Veiga-Borgeaud et al.(13), que afirmaram que o ritmo de reprodução desses roedores é máximo durante a estação chuvosa e menos intenso, ou praticamente nulo, durante a estação seca.

Esse resultado concorda também com os do estudo realizado no Pamparrão, Rio de Janeiro, onde Nectomys squamipes, roedor silvestre também atuante como reservatório da esquistossomose, apresentou reprodução o ano inteiro, com predominância nos períodos chuvosos devido à sua associação com recursos encontrados na água. Nas populações do roedor, a sobrevivência e o recrutamento foram maiores no final da época chuvosa. A reprodução seria desencadeada pela disponibilidade dos recursos, resultando em aumento nas taxas de sobrevivência após os períodos de chuva ${ }^{(30)}$.

Assim como o Nectomys squamipes, o motivo para a reprodução do roedor Holochilus sp. se intensificar 
na época de chuvas provavelmente é decorrente também da maior disponibilidade de recursos encontrada nessa época, pois sabe-se que a vegetação aquática fica mais abundante, proporcionando alimento, local para construir o ninho e até facilidade para se esconder de predadores.

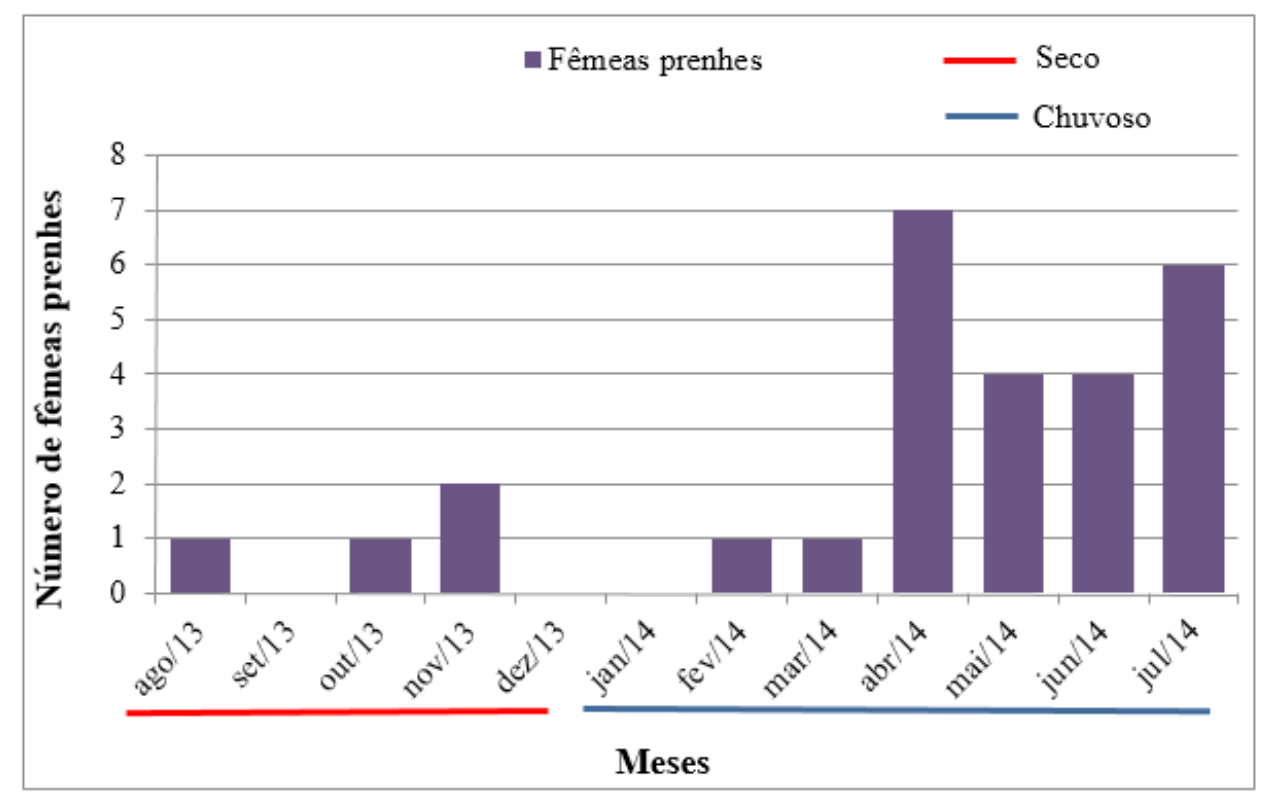

Figura 2: Fêmeas prenhes encontradas nos períodos de seca e chuva em São Bento - MA.

Em relação à positividade para $S$. mansoni, observou-se que houve quase que uma constância da infecção para o período trabalhado, à exceção do mês de julho, em que não foi encontrado nenhum roedor infectado. O mês de maio apresentou o maior índice de positividade e, dos oito roedores capturados, 55,5\% estavam infectados com a parasitose. Em novembro, fevereiro e março, encontrouse um percentual de 30\% de infecção nos animais. No mês de setembro, constatou-se um menor índice de infecção, com apenas $10 \%$, porém foi um dos meses em que se obteve um menor número de roedores (Figura 3).

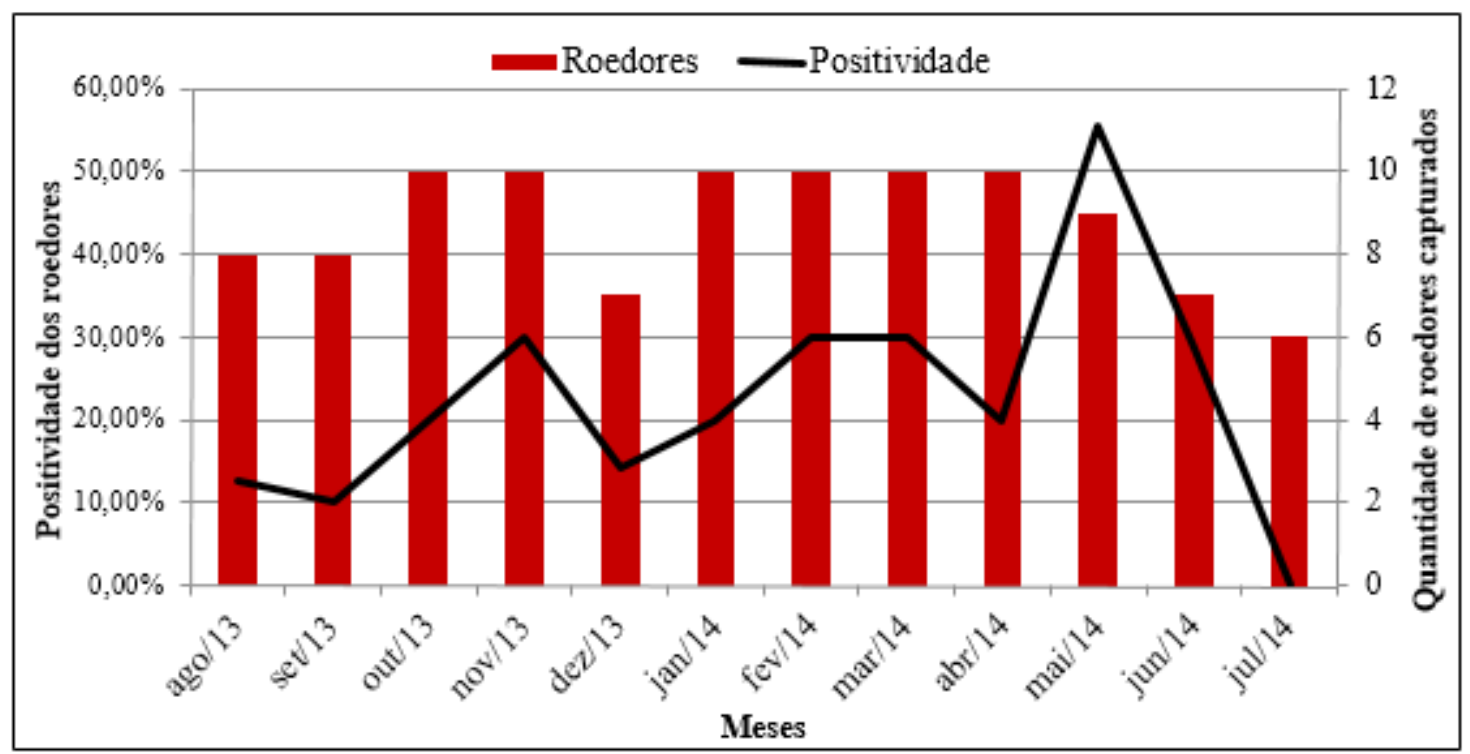

Figura 3: Positividade para S. mansoni dos roedores silvestres, por mês, no município de São Bento - MA. 
A captura realizada mensalmente no município de São Bento confirmou a importância dos roedores silvestres como elemento portador e difusor da esquistossomose mansônica na região da Baixada Maranhense, visto que foram encontrados Holochilus sp. liberando ovos viáveis nas fezes. Miranda et al. ${ }^{(31)}$ obtiveram resultado semelhante para positividade de roedores Holochilus sp. na Baixada Maranhense, onde os animais demonstraram alto grau de infecção natural por S. mansoni e eliminaram ovos em suas fezes em praticamente todos os meses do período trabalhado pelos autores.

Segundo Gentile et al. ${ }^{(6)}$, em condições naturais, a infecção por $S$. mansoni não afeta a sobrevivência, capacidade de reprodução e a mobilidade de roedores (Nectomys sp.), permitindo a transmissão do parasito ao longo de toda a sua vida. Além disso, como os Holochilus sp. possuem hábito semiaquático, eles conseguem facilmente se infectar com cercárias de $S$. mansoni, completando, assim, o ciclo do parasito. O contato com a água também facilita a eclosão dos ovos viáveis do helminto, eliminados nas fezes dos roedores, possibilitando a infecção do hospedeiro intermediário (caramujo do gênero Biomphalaria), o qual posteriormente poderá infectar tanto hospedeiros definitivos humanos, principalmente pescadores que constantemente entram em contato com os campos alagados de São Bento, como também outros roedores, incluindo os filhotes.

É importante ressaltar, também, que a positividade para $S$. mansoni e os dados reprodutivos encontrados para o roedor do gênero Holochilus mostraram-se mais intensos no mesmo período no município de São Bento - MA (época de chuvas). Isso é bastante preocupante, visto que esses animais são eficientes reservatórios para a doença e, dessa maneira, a alta reprodução contribui ainda mais para aumentar esse índice, pois, à medida que esses animais nascem e o ciclo da esquistossomose se mantém próximo a eles, maior a probabilidade de aumentar o número desses potenciais reservatórios naturais da doença.

\section{Conclusão}

O número de fêmeas capturadas foi menor do que o de machos, sendo eles maiores e mais pesados do que as fêmeas. Os roedores do gênero Holochilus encontrados mostraram-se suscetíveis à infecção por $S$. mansoni e as fêmeas prenhes capturadas apontaram que esse animal tem um alto potencial reprodutivo. Além disso, o período chuvoso do município de São Bento - MA correspondeu ao de maior intensidade reprodutiva para os roedores do gênero Holochilus, pois eles possuem hábito semiaquático e dependem de recursos da época de chuvas para a obtenção de energia para se reproduzir com maior eficiência. A alta taxa de infecção e a intensa atividade reprodutiva dos roedores, só agravam a esquistossomose na Baixada Maranhense, pois aumenta o número de roedores de possíveis reservatórios da esquistossomose e, consequentemente, contribui para um aumento da probabilidade de infecção em humanos.

\section{Agradecimentos}

À Fundação de Amparo à Pesquisa e ao Desenvolvimento Científico e Tecnológico do Maranhão (FAPEMA) e a Universidade Estadual do Maranhão (UEMA), pelo auxílio financeiro.

\section{Referências}

1. World Health Organization (WHO). Sustaining the drive to overcome the global impact of neglected tropical diseases: second WHO report on neglected tropical diseases. Geneva: World Health Organization. 2013. Disponivel em http://www.who.int/iris/bitstream/10665/77950/1/9789241564540 eng.pdf. Acesso em junho de 2015.

2. Nascimento GL, Oliveira MR. Severe forms of schistosomiasis mansoni: epidemiologic and economic impact in Brazil, 2010. Trans R. Soc. Trop. Med. Hyg. 2014 Dec [citado 2015 nov 29]; 108(1)29-36. Disponível em: http://trstmh.oxfordjournals.org/content/108/1/29.abstract. Inglês.

3. Delmondes LM, Cruz MA, Guimarães MK, Santana LG, Gonçalves VP, Brito HL. Esquistossomose 
endocervical: relato de caso. Rev Bras Ginecol Obstet. 2014 Jun [citado 2015 jun 08]; 36(6)276-80. Disponível em: http://dx.doi.org/10.1590/S0100-720320140004827.

4. Despres L, Imbert-Establet D, Combes C, Bonhomme F., E. Molecular evidence linking hominid evolution to recent radiation of Schistosome (Platyhelminths: Trematoda). Mol. Phylogenet. Evol. 1992 Dec [citado 2015 Jun 08]; 1(4):295-304. Disponível em: http://www.researchgate.net/publication/21812655_Molecular_Evidence Linking_Hominid_Evolution_to_Recent_Radiation_of_Schistosomes_(Platyhelminthes_Trematoda). Inglês.

5. Morgan JA, DeJong RJ, Kazibwe F, Mkoji GM, Loker ES. A newly - identified lineage of Schistosoma. Int. J. Parasitol. 2003 Set [citado 2015 jun 08]; 33(9):977- 985. Disponível em: http://dx.doi.org/10.1016/S00207519(03)00132-2. Inglês.

6. Gentile R, Neto SFN, D'andrea PS. Uma revisão sobre a participação do rato d'água Nectomys squamipes na dinâmica de transmissão da esquistossomose mansônica: um estudo multidisciplinar de longo prazo em uma área endêmica. Rev. Oecologia Australis. 2010 [citado 2015 jun 08]; 14(3):711-725. Disponível em: http:// www.oecologiaaustralis.org/ojs/index.php/oa/article/view/oeco.2010.1403.07.

7. Kerber L, Lopes RP, Oliveira EV, Ribeiro AM, Pereira J. On the presence of Holochilus brasiliensis (Desmarest, 1819) (Rodentia: Cricetidae: Sigmodontinae) in the late Pleistocene. Journal of Geoscience. 2010 [citado 2015 jun 08]; 8(2):47-54. Disponível em: file://C:/Users/User/Downloads/7613-23172-1-PB.pdf. Inglês.

8. Bonvicino CR, Oliveira JA, D'Andrea PS. Guia dos roedores do Brasil, com chaves para gêneros baseadas em caracteres externos. Rio de Janeiro: Centro Pan-Anamericano de Febre Aftosa - OPAS/OMS; 2008. 120 p. Disponível em: http://www.fiocruz.br/ioc/media/livro\%20roedores.pdf. Acesso em junho de 2015.

9. Gentile R, Costa-Neto SF, Gonçalves MML, Bonecker ST, Fernandes FA, Garcia JS, Barreto MGM, Soares MS, D'Andrea PS, Peralta JM, Rey L. An ecological field study of the water-rat Nectomys squamipes as a wild reservoir indicator of Schistosoma mansoni transmission in an endemic area. Mem. Inst. Oswaldo Cruz. 2006 [citado 2015 jun 08]; Vol. 101(Suppl. I):111-117. Disponível em http://dx.doi.org/10.1590/S007402762006000900018. Inglês.

10. Ernest KA, Mares MA. Ecology of Nectomys squamipes, the Neotropical Water rat, in central Brazil: home range, habitat selection, reproduction and behavior. J. Zool. 1986 Dez [citado 2015 jun 08]; 210(4):599-612. Disponível em: http://dx.doi.org/10.1111/j.1469-7998.1986.tb03658.x. Inglês.

11. Mello DA. Breeding of wild-caught rodent cricetidae Holochilus brasiliensis under laboratory conditions. Lab. Anim. 1986 Jul 01 [citado 2015 jun 08]; 20(3):195-196. Disponível em: http://lan.sagepub.com/ content/20/3/195.long. Inglês.

12. Bastos OC. Holochilus brasiliensis nanus Thomas, 1987. Sugestão de modelo experimental para filariose, lesihmaniose e esquistossomose. Rev. Inst. Méd. Trop, São Paulo. 1984 Nov [citado 2015 jun 08]; 26(4):307315. Disponível em: http://www.scielo.br/pdf/rimtsp/v26n6/04.pdf.

13. Veiga-Borgeaud T, Neto RCL, Peter F, Bastos OC. Constatações sobre a importância dos roedores silvestres (Holochilus brasiliensis nanus. Thomas, 1981) na epidemiologia da esquistossomose própria da Pré-Amazônia. Cad. Pesq. 1986 Jul [citado 2015 jun 08]; 2(1):86-99. Disponível em: http://www.pppg.ufma. br/cadernosdepesquisa/uploads/files/Artigo\%201(4).pdf.

14. Cantanhede SPD, Fernandez MA, Mattos AC, Montresor LC, Silva-Souza N, Thiengo SC. Freshwater gastropods of the Baixada Maranhense Microregion, an endemic area for schistosomiasis in the State of Maranhao, Brazil: I - qualitative study. Rev. Soc. Bras. Med. Trop. 2014 Jan [citado 2015 jun 08];47(1):79-85. Disponível em: http://www.scielo.br/pdf/rsbmt/v47n1/0037-8682-rsbmt-47-01-79.pdf.

15. Ozanan CCAF. Notas sobre o rato de cana, "Holochilus sciureus" Wagner, na região do Cariri, Ceará. Ver. Bras. Biol. 1969 [citado 2015 jun 08]; 29(4):567-570. Disponível em: http://www.scielo.br/scielo. php?script $=$ sci nlinks\&ref $=8487524 \&$ pid $=S 0101-8175199700050000100182 \& \operatorname{lng}=$ en.

16. Sauthier WOU, Abba AM, Sauthier DEU. Nests of Oligoryzomys sp. an Holochilus brasiliensis (Rodentia, Cricetidae) eastern Entre Rios Province, Argentina. Mastozool.neotrop. 2010 Jan [citado 2015 jun 08]; 17(1):207-

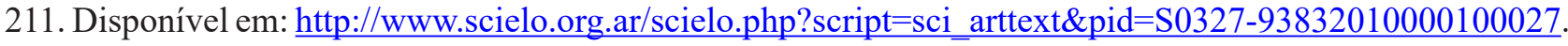


Inglês.

17. Katz N, Chaves A, Pellegrino J. A simple device for quantitative stool thick-smear technique in Schistosomiasis manson. Rev. Inst. Méd. Trop, São Paulo. 1972 Nov [citado 2015 jun 08]; 14(6):397-400. Disponível em: http://www.researchgate.net/publication/18489942_A_simple_device_for_quantitative stool thick-smear_in_Schistosoma_mansoni. Inglês.

18. Paixão RL. Bioética e medicina veterinária: um encontro necessário. Revista do Conselho Federal de Medicina Veterinária, ano 7, 2001 [citado 2015 jun 08]; 23:20-26, 2001. Disponível em: http://www.fcav. unesp.br/Home/departamentos/patologia/ANTONIOCARLOSALESSI/bioetica_e med vet.pdf.

19. Zar JH. Biostatistical analysis. 5th ed. New Jersey: Prentice-Hall International INC; 2010. 960p. Inglês.

20. Giarrizzo T, Silva de Jesus AJ, Lameira EC, Araújo de Almeida JB, Isaac V, Saint-Paul U. Weight-length relationships for intertidal fauna in a mangrove estuary in Northern Brazil. J. Appl. Ichthyol. 2006 [citado 2015 jun 08]; 22(4):325-327. Disponível em: http://www.researchgate.net/publication/229056103 Weightlength relationships_for_intertidal_fish_fauna in_a_mangrove estuary_in_Northern_Brazil. Inglês.

21. Graipel ME, Cherem JJ, Monteiro-Filho ELA, Glock L. Dinâmica populacional de marsupiais e roedores no Parque Municipal da Lagoa do Peri, Ilha de Santa Catarina, sul do Brasil. Mastozool. Neotrop. 2006 Jan [citado 2015 jun 08]; 13(1): 31-49. Disponível em: http://www.scielo.org.ar/scielo.php?script=sci arttext\&pid=S0327-93832006000100004.

22. Cherem JJ, Simões-Lopes PC, Althoff S, Graipel ME. Lista dos mamíferos do estado de Santa Catarina, sul do Brasil. Mastozoología Neotropical. 2004 [citado 2015 jun 08]; 11(2):151-184. Disponível em: http://www. scielo.org.ar/scielo.php?script=sci arttext\&pid=S0327-93832004000200002.

23. Graipel ME, Santos-Filho M. Reprodução e dinâmica populacional de Didelphis aurita Wied-Neuwied (Mammalia: Didelphimorphia) em ambiente periurbano na Ilha de Santa Catarina, Sul do Brasil. Biotemas. 2006 [citado 2015 jun 08]; 19:65-73. Disponível em: https://periodicos.ufsc.br/index.php/biotemas/article/ view/21331/19240.

24. D' andrea PS, Gentile RI, Maroja LS, Fernandes FA, Coura R, Cerqueira RV. Small mammal populations of an agroecosystem in the Atlantic Forest domain, southeastern Brazil. Braz. J. Biol. 2007 Fev [citado 2015 jun 08]; 67(1):179-186. Disponível em: http://dx.doi.org/10.1590/S1519-69842007000100025. Inglês.

25. Bergallo HG, Magnusson WE. Effects of climate and food availability on four rodent species in southeastern Brazil. J. Mammal. 1999 May [citado 2015 nov 29]; 80(2)472-486. Disponível em: http://www.researchgate. net/publication/267508423 Effects_of_Climate_and_Food_Availability_on_Four_Rodent_Species in Southeastern_Brazil. Inglês

26. Bergallo HG, Magnusson WE. Factors affecting the use of space by two rodent species in Brazilian Atlantic forest. Mammal. 2004 [citado 2015 jun 08]; 68(2-3):121-132. Disponível em: http://www.researchgate. net/publication/249944117 Factors affecting the use of space by two rodent species in Brazilian Atlantic forest. Inglês.

27. Bergallo HG. Ecology of a small mammal community in an Atlantic Forest area of South-eastern Brazil. Stud. Neotrop. Fauna \& Environ. 1994 [citado 2015 nov 29]; 29(4)197-217. Disponível em: http://www. researchgate.net/publication/233330041_Ecology of a small mammal_community in an Atlantic_Forest area in_Southeastern_Brazil. Inglês.

28. D'Andrea OS, Horta C, Cerqueira R, Rey L. Breeding of the water rat (Nectomys squamipes) in the laboratory. Lab. Anim. 1996 Jan [citado 2015 jun 08]; 30(4):369-376. Disponível em: http://lan.sagepub.com/ content/30/4/369.full.pdf. Inglês.

29. Kawazoe U, Pinto ACM. Importância epidemiológica de alguns animais silvestres na esquistossomose mansônica. Rev. Saúde Publ., São Paulo. 1983 Out [citado 2015 jun 08]; 17(5):345-66. Disponível em: http:// dx.doi.org/10.1590/S0034-89101983000500001.

30. Bonecker ST, Portugal LG, Costa-Neto SF, Gentile R. A long term study of small mammal populations in a Brazilian agricultural landscape. Mammal. Biol. 2009 Nov [citado 2015 jun 08]; 74( 6):467-477, 2009. Disponível em: http://dx.doi.org/10.1016/j.mambio.2009.05.010. Inglês. 
31. Miranda GS, Rodrigues JGM, Lira MGS, Nogueira RA, Gomes GCC, Silva-Souza N. Monitoramento de positividade para Schistosoma mansoni em roedores Holochilus sp. naturalmente infectados. Cienc. anim. bras. 2015 Jul [citado 2015 ago 02]; 16(3):456-463. Disponível em: http://www.revistas.ufg.br/index.php/vet/ article/view/33164/18837. 